\title{
Prediction of Cirrhosis in Patients with Chronic Hepatitis C by Genotype 3
}

\author{
Tarana Gupta ${ }^{1}$, Hari K Aggarwal ${ }^{2}$, Sandeep Goyal ${ }^{3}$, Virendra Singh ${ }^{4}$
}

\begin{abstract}
Background: Genotype 3 increases fibrosis in chronic hepatitis C (CHC). Aim: To evaluate the effect of the hepatitis C virus (HCV) genotype on prevalence and severity of liver disease in CHC. Materials and methods: Nine hundred and forty-nine individuals with positive anti-HCV from June 2016 to May 2017 were enrolled in the study. We compared biochemical and hematological parameters, HCV RNA load, transient elastography, and ultrasound, in genotype 3 and nongenotype 3 patients. Cirrhosis was diagnosed in patients with liver stiffness measurement (LSM) $\geq 13 \mathrm{kPa}$.

Results: Out of 835 CHC patients, overall, genotype 3 had higher LSM (11.3 vs 7.62, $p=0.01$ ), higher aspartate aminotransferase (AST) (88.4 vs $68.6, p=0.02)$, and low platelets (228.4 vs $261, p=0.03)$ with higher prevalence of cirrhosis $(115 / 415$ vs $25 / 245, p=0.01)$ than nongenotype 3 . However, decompensation rates were not significantly different between two groups $(32 / 115 \mathrm{vs} 7 / 25, p=0.98)$. The subgroup analysis revealed that cirrhotic genotype 3 had advanced age ( 50 vs $35, p<0.01$ ), male predominance, and higher AST $(74.4$ vs $57, p=0.01$ ) as compared to noncirrhotic genotype 3 patients. On multivariate analysis, age and AST values were higher in cirrhotic than noncirrhotic genotype 3 patients. Conclusion: Genotype 3 patients have higher prevalence of cirrhosis and fibrosis compared to nongenotype 3 patients; however, decompensation was not different between two groups.

Keywords: Chronic hepatitis C, Cirrhosis, Fibrosis, Genotype, Transient elastography.

Euroasian Journal of Hepato-Gastroenterology (2020): 10.5005/jp-journals-10018-1311
\end{abstract}

\section{INTRODUCTION}

Chronic hepatitis $\mathrm{C}(\mathrm{CHC})$ is an important cause of chronic liver disease, cirrhosis, hepatocellular carcinoma (HCC), and liver disease-related mortality. In the era of direct-acting antivirals (DAAs) with their excellent efficacy and safety, hepatitis $C$ virus (HCV) infection still remains an important cause of chronic liver disease as these patients are largely asymptomatic till they develop significant fibrosis and cirrhosis. Various studies have evaluated the risk factors responsible for progression of fibrosis in chronic hepatitis C patients. ${ }^{1,2}$ These may be modifiable risk factors like alcohol consumption, cigarette smoking, hepatitis $B$ and human immunodeficiency virus (HIV) coinfection, presence of obesity, diabetes mellitus, and metabolic syndrome. On the other hand, nonmodifiable risk factors like age at acquiring infection, sex, race, and HCV genotype have also been noted to influence rate of fibrosis progression. ${ }^{3}$ In earlier studies, it was observed that genotype 1 has faster rate of fibrosis progression than other genotypes. ${ }^{4}$ Later, Probst et al. in their meta-analysis showed a faster liver fibrosis progression rate in genotype 3 patients in single biopsy studies. ${ }^{5}$ Large retrospective and prospective data analysis of U.S. veterans has shown that genotype 3 is associated with increased risk of developing cirrhosis and HCC. ${ }^{6}$

With availability of DAAs as more effective and safer therapies, the goals in HCV management have shifted from treatment of individual patients toward HCV eradication and to achieve this, large community-based programs are being undertaken in India. In these programs, the treatment of chronic hepatitis $C$ is aimed to target maximum number of HCV-infected individuals. Although, the combination therapy of sofosbuvir and daclatasvir has a good efficacy in noncirrhotic chronic hepatitis $C$ patients, the efficacy in genotype 3 cirrhotic patients is less. Also, as more complete data of DAAs have emerged, it has become apparent that genotype 3

\begin{abstract}
1,2Department of Medicine, Post Graduate Institute of Medical Sciences, Pandit Bhagwat Dayal Sharma University of Health Sciences, Rohtak, Haryana, India

${ }^{3}$ Himachal Pradesh, India

${ }^{4}$ Department of Hepatology, Postgraduate Institute of Medical Education and Research, Chandigarh, India

Address reprint requests to: Tarana Gupta, Department of Medicine, Post Graduate Institute of Medical Sciences, Pandit Bhagwat Dayal Sharma University of Health Sciences, Rohtak, Haryana, India, Phone: +919914048899, e-mail: taranagupta@gmail.com

How to cite this article: Gupta T, Aggarwal HK, Goyal S, et al. Prediction of Cirrhosis in Patients with Chronic Hepatitis C by Genotype 3. Euroasian J Hepato-Gastroenterol 2020;10(1):7-10.

Source of support: Nil

Conflict of interest: None
\end{abstract}

has a lower sustained virological response (SVR) rate than other genotypes especially in patients with cirrhosis and is emerging as a new treatment challenge. The present study has aimed to evaluate the effect of the hepatitis $C$ genotype on prevalence and severity of liver disease in chronic hepatitis $C$ patients.

\section{Materials and Methods}

It was a cross-sectional study, conducted at Post Graduate Institute of Medical Sciences, Pandit Bhagwat Dayal Sharma University of Health Sciences, Rohtak, Haryana, India. All the patients aged $>18$ years with newly diagnosed chronic hepatitis $C$ infection attending the Hepatobiliary Clinic of Department of Medicine from June 2016 to May 2017 were included into the study after obtaining a written informed consent. Patients with age $<18$ years, history of alcohol intake $>40 \mathrm{~g} /$ day, chronic hepatitis B, HIV infection, diabetes 
mellitus, pregnancy, comorbid conditions like cardiovascular disease, chronic renal disease, autoimmune diseases, HCC, and nonhepatic malignancies, and patients on immunosuppressive and cytotoxic therapy were excluded. Chronic hepatitis $\mathrm{C}$ infection was diagnosed by detecting anti-HCV antibodies by enzyme-linked immunosorbent assay (ELISA). Hepatitis $C$ infection was further evaluated with HCV RNA polymerase chain reaction (PCR) and the HCV genotype. History, physical examination, hematological and biochemical investigations like hemogram, liver function tests, prothrombin time and renal function tests, ultrasonography (USG), and transient elastography (TE) were done.

The TE values in patients with ascites, elevated liver enzymes $>5$ ULN, and elevated bilirubin were not included into the study. The value of TE was expressed as median of minimum 10 readings of liver stiffness measurement (LSM) with interquartile range (IQR) $<30 \%$.

As per Castera et al., ${ }^{7-9}$ cirrhosis was diagnosed with the LSM value $>13 \mathrm{kPa}$ in a patient or if elastography was contraindicated, then based on USG findings, i.e., nodular margins of liver, shrunken liver size, features suggestive of portal hypertension like portal vein diameter $>14 \mathrm{~mm}$, splenomegaly, presence of collaterals, and ascites. ${ }^{10,11}$ Patients with cirrhosis (as defined by LSM values and/or USG findings) were evaluated for decompensation of liver disease such as presence of jaundice, ascites, upper gastrointestinal bleed, and hepatic encephalopathy. All patients were grouped into genotype 3 and nongenotype 3 . All biochemical parameters and TE values were compared among both the groups.

\section{Statistical Analysis}

The quantitative variables were presented as mean with standard deviation/range and qualitative variables as proportions with percentages. All parametric and nonparametric variables were compared using the Student's $t$ test and the Mann-Whitney $U$ test, respectively. The categorical variables were compared using the chi-square test and the Fisher exact test. The $p$ value less than 0.05 was taken as significant. All the variables with $p<0.1$ in the univariate analysis were included into the multivariate analysis by the backward conditional logistic regression analysis. The statistical analysis was performed using SPSS v20.

\section{ResULTS}

Total 949 individuals were found to be anti-HCV antibody positive by ELISA. Out of these, 843 patients had detectable HCV RNA by PCR and remaining 106 patients had undetectable HCV RNA (Flowchart 1). Seven patients had coinfection with hepatitis $B$, one had HIV coinfection and were excluded from the study. Among 835 patients, 542 had genotype 3 and 293 had nongenotype 3 . Out of 835 patients, LSM values were available in 618 patients, which included 387 patients of genotype 3 and 231 patients of nongenotype 3 . Transient elastography either failed or was contraindicated in remaining 217 patients due to presence of obesity, ascites, raised transaminases, or high bilirubin.

The mean LSM values among genotype $3(n=387)$ and nongenotype $3(n=281)$ were $11.36 \mathrm{kPa}$ and $7.62 \mathrm{kPa}(p<0.001)$, respectively. Out of 618 patients, 111 had cirrhosis as diagnosed by LSM values $>13 \mathrm{kPa}$. In remaining noncirrhotic patients $(n=507)$, the mean LSM values were higher in the genotype 3 group $(n=$ 296) than the nongenotype 3 group $(n=211)(6.75 \mathrm{kPa}$ vs $6.11 \mathrm{kPa}$, $p<0.001$ ), respectively (Table 1 ).

In 225 patients in whom TE could not be done, 68 patients had cirrhosis as diagnosed by ultrasound findings. Out of these 68
Flowchart 1: Flow of patients into the study

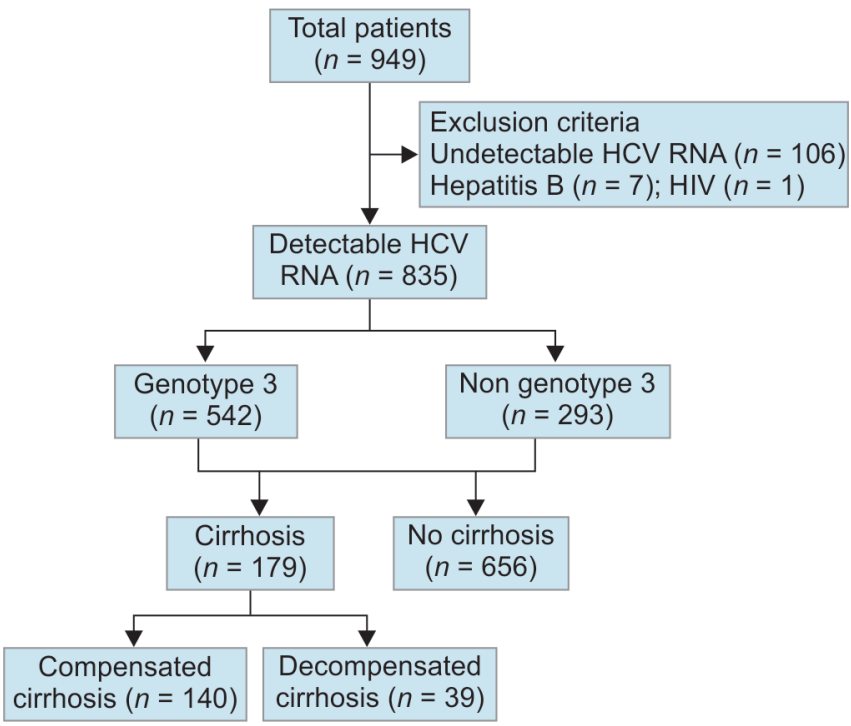

Table 1: Liver stiffness measurement $(\mathrm{kPa})$ comparison between genotype 3 and nongenotype 3 patients

\begin{tabular}{llll}
\hline & ${\text { Genotype } 3^{+}}^{+}$ & Nongenotype $^{+}$ & pvalue \\
\hline All $(n=618)$ & $11.36(3.4-49.7)$ & $7.62(3.1-35.3)$ & $<0.01$ \\
& $(n=387)$ & $(n=231)$ & \\
Non-cirrhotic & $\begin{array}{l}6.75(3.4-12.2) \\
(n=507)\end{array}$ & $\begin{array}{l}6.11(2.7-11.8) \\
(n=296)\end{array}$ & $<0.01$ \\
\hline
\end{tabular}

${ }^{\dagger}$ Based on LSM

patients, 29 had compensated cirrhosis and 39 had decompensated cirrhosis due to presence of either ascites, upper gastrointestinal bleed, hepatic encephalopathy, or jaundice.

A total of 179 patients had cirrhosis with 140 having compensated cirrhosis and decompensation present in 39 patients. Among 140 patients with compensated cirrhosis, 115 were genotype 3 and remaining 25 were nongenotype 3 (Table 2). Out of 39 patients with decompensated cirrhosis, 32 had genotype 3 and 7 had nongenotype 3 (Table 2). Overall, genotype 3 had higher prevalence of cirrhosis than nongenotype 3 ( $115 / 415$ vs $25 / 245, p=0.01$ ). However, decompensation rates were not different between two groups (32/115 vs $7 / 25, p=0.98)$.

Overall, the genotype 3 patients as compared to nongenotype 3 patients had low platelet count ( 228.4 vs $261.9, p=0.03$ ) and high aspartate aminotransferase (AST) values (88.4 U/L vs $68.6 \mathrm{U} / \mathrm{L}, p=$ 0.02 ), respectively (Table 3 ). In the subgroup analysis, the cirrhotic genotype 3 patients had more advanced age (50 vs $35, p \leq 0.01$ ), male predominance, higher AST values (74.4 U/L vs $57.7 \mathrm{U} / \mathrm{L}, p=$ 0.01 ), and low platelet counts ( 241.1 vs $263.6, p=0.16$ ), respectively (Table 3), as compared to noncirrhotic genotype 3 patients. On multivariate analysis, age and AST values were significantly higher in cirrhotic than noncirrhotic genotype 3 patients.

\section{Discussion}

Our study showed that genotype 3 is the most common genotype in northern India. The genotype 3 is associated with higher prevalence of cirrhosis as shown by higher LSM values and ultrasound findings. Overall, genotype 3 patients have advanced fibrosis as suggested by higher LSM values, low platelet counts, and higher AST values as 
Table 2: Prevalence of compensated and decompensated cirrhosis between genotype 3 and nongenotype 3 patients

\begin{tabular}{llllll}
\hline & ${\text { No } \text { cirrhosis }^{a}}$ & Cirrhosis $^{b}$ & Decompensated cirrhosis $^{c}$ & $p^{a b}$ value & $p^{b c}$ value \\
\hline Genotype 3 & 300 & 115 & 32 & $<0.01$ & 0.98 \\
Nongenotype 3 & 220 & 25 & 7 & & \\
\hline
\end{tabular}

Cirrhosis was diagnosed by either TE or USG findings

$p^{\mathrm{ab}}=$ significant difference between no cirrhosis and cirrhosis

$p^{\mathrm{bc}}=$ significant difference between cirrhosis and decompensated cirrhosis

Table 3: Baseline characteristics

\begin{tabular}{|c|c|c|c|c|c|c|c|}
\hline \multirow[b]{2}{*}{ Variable } & \multirow[b]{2}{*}{ Genotype 3} & \multirow[b]{2}{*}{ Nongenotype 3} & \multirow[b]{2}{*}{ pvalue } & $\begin{array}{l}\text { Genotype } 3 \\
\text { (cirrhotic) }\end{array}$ & $\begin{array}{l}\text { Genotype } 3 \\
\text { (noncirrhotic) }\end{array}$ & & \multirow{2}{*}{$\begin{array}{l}\text { Multivariate } \\
\text { analysis }\end{array}$} \\
\hline & & & & \multicolumn{3}{|c|}{ Univariate analysis } & \\
\hline Age (years) & $40(5-85)$ & $38(10-87)$ & 0.69 & $50(19-85)$ & $35(5-76)$ & $<0.01$ & $<0.01$ \\
\hline Sex $(M: F)$ & $1.2: 1$ & $1.1: 1$ & 0.76 & $1.8: 1$ & $1.1: 1$ & $<0.01$ & - \\
\hline $\mathrm{Hb}(\mathrm{g} \%)$ & $13.5(6.9-17.3)$ & $13.8(8-18.2)$ & 0.24 & $13.7(7.5-17.3)$ & $13.9(8-18.3)$ & 0.51 & - \\
\hline $\operatorname{TLC}\left(\times 10^{3} / \mathrm{mm}^{3}\right)$ & $7.4(5.5-22)$ & $7.4(1-12)$ & 0.91 & $7.4(5.5-14)$ & $7.5(1-17)$ & 0.64 & - \\
\hline Platelets $\left(\times 10^{3} / \mathrm{L}\right)$ & $228.4(60-700)$ & $261(80-900)$ & 0.03 & $241.1(60-500)$ & $263.6(180-550)$ & 0.16 & - \\
\hline AST (U/L) & $88.4(6-354)$ & $68.6(22-350)$ & 0.02 & $74.4(6-334)$ & $57(3.1-257)$ & 0.01 & $<0.01$ \\
\hline $\operatorname{ALT}(\mathrm{U} / \mathrm{L})$ & $115.6(6.14-614)$ & $99.8(15-628)$ & 0.24 & $108.5(6.4-515)$ & $88(15-442)$ & 0.10 & - \\
\hline ALP & $96.9(2.07-326)$ & $92.5(35-226)$ & 0.45 & $88.6(39-326)$ & $87.3(35-187)$ & 0.79 & - \\
\hline Albumin (g/dL) & $4(1.5-6.70)$ & $4.2(0.7-6.96)$ & 0.16 & $5.9(1.5-6.9)$ & $6.7(1.5-8.4)$ & 0.38 & - \\
\hline PT & $14.8(10.3-28.7)$ & $14.7(10-59.5)$ & 0.83 & $14.1(10.3-30.9)$ & $14.4(10-59.5)$ & 0.66 & \\
\hline
\end{tabular}

Data presented as median (IQR); LSM, liver stiffness measurement; $\mathrm{Hb}$, hemoglobin; TLC, total leukocyte count; AST, aspartate aminotransferase; ALT, alanine aminotransferase; ALP, alkaline phosphatase; PT, prothrombin time

compared to nongenotype 3 patients. Even in cirrhotic genotype 3 patients, advanced age with male predominance and higher AST values are observed than noncirrhotic genotype 3 patients. The multivariate analysis showed that age and AST values are higher in cirrhotic than noncirrhotic genotype 3 patients.

Lehman et al. showed that genotype 3 prevalence was higher among individuals who had spontaneously cleared HCV infection as compared to patients with chronic infection ( $86 \%$ vs $38 \%$; $p=0.002$ ). Moreover, the chronic infection was noted in higher number of individuals exposed to genotype 1 infection as compared to genotype 3 (93\% vs 63\%, $p=0.006) .{ }^{12}$ In the interferon era, genotype 3 had higher SVR rates as compared to other genotypes. It was suggested that perhaps genotype 3 induces higher interferon gene transcription in monocyte-macrophages and dendritic cell lines, which leads to increased immune clearance. At the same time, this enhanced interferon gene stimulation in nonparenchymal cells of the liver fastens the rate of fibrosis. ${ }^{13}$

In addition, HCV genotype 3 has been shown to be associated with hepatic steatosis and it is independent of presence of obesity, high body mass index, diabetes mellitus, age, and hepatic inflammation. ${ }^{14}$ Ryan et al. ${ }^{15}$ showed that genes of lipogenesis are not overexpressed in liver biopsies of hepatitis C-infected patients. In fact, the hepatic steatosis is due to overexpression of chemokines, which leads to increased stimulation of inflammatory cells within the liver. The association of genotype 3 and hepatic steatosis is suggested by its association with higher HCV viral load, presence of steatosis even in absence of metabolic risk factors, and the resolution of steatosis after SVR has been achieved post antiviral treatment. ${ }^{16,17}$ It has been suggested that in genotype 3 some genes like core protein are responsible for additional cytopathic effect, which morphologically present as hepatic steatosis. ${ }^{18}$
Bochud et al. ${ }^{19}$ also found faster progression of fibrosis in genotype 3 patients who were intravenous drug abusers as compared to those who acquired HCV infection through blood transfusion or needle injuries. De Nicola et al. ${ }^{20}$ showed lower mean time lag necessary for development of significant fibrosis in genotype 3 as compared to genotype 1 (28.3 years vs 21.8 years, $p=0.03$ ), respectively. The enhanced interferon gene transcription, higher viral load, and hepatic steatosis are some mechanisms that may be responsible for fastened liver fibrosis, cirrhosis, and development of HCC.

In the present study, we found that genotype 3 is the most common genotype in northern India and it is more commonly associated with advanced fibrosis as suggested by higher LSM values. We also demonstrated that platelet counts are lower in genotype 3 patients as compared to other genotypes along with higher LSM values indicating higher stages of fibrosis in genotype 3 patients. However, once the cirrhosis has established, the decompensation rates were similar between genotype 3 and nongenotype 3 patients. The DAAs can be used in decompensated cirrhosis; however, not all patients improve clinically after treatment. Therefore, early diagnosis and treatment is essential to improve overall liver-related mortality. Further, advanced age and higher AST values were noted in cirrhotic genotype 3 patients than noncirrhotic genotype 3 patients.

The present study has some limitations. The liver biopsy is a gold standard for diagnosis of cirrhosis; however, it is an invasive procedure that is associated with complications like pain, bleeding, and mortality. Therefore, we have taken TE or ultrasonography as the diagnostic criteria for diagnosis of cirrhosis. The exact duration of HCV infection could not be determined in a majority of the patients due to poor socioeconomic and educational status and 
hence suboptimal history; however, this is a cross-sectional study and demonstrates increased prevalence of advanced fibrosis and cirrhosis in genotype 3 chronic hepatitis $C$ patients compared to nongenotype $3 \mathrm{CHC}$ patients, thus reflecting higher rates of progression in genotype $3 \mathrm{CHC}$ patients. In future, we need more prospective studies to evaluate the impact of genotype on development of cirrhosis and HCC and also whether subgenotypes are also responsible. In the era of DAAs, when genotype 3 is emerging as a new treatment challenge, it is important to elucidate whether control of other metabolic and confounding factors can improve the overall outcome in these patients.

Genotype 3 patients have higher prevalence of fibrosis and cirrhosis indicating more severe liver disease than other genotypes. In our part of the world, genotype 3 is the predominant genotype. Advanced age, male sex, higher AST values, lower platelet counts, and higher LSM values should be carefully assessed in these patients for diagnosis of cirrhosis. The HCV treatment with DAAs does not reverse the cirrhosis and advanced fibrosis completely, and these patients need to be followed up regularly for development of decompensation and HCC.

\section{References}

1. Massard J, Ratziu V, Thabut D, et al. Natural history and predictors of disease severity in chronic hepatitis C. J Hepatol 2006; 44(1 Suppl):S19-S24. DOI: 10.1016/j.jhep.2005.11.009.

2. Rüeger S, Bochud PY, Dufour JF, et al. Impact of common risk factors of fibrosis progression in chronic hepatitis C. Gut 2015;64(10):1605-1615. DOI: 10.1136/gutjnl-2014-306997.

3. Missiha SB, Ostrowski M, Heathcote EJ. Disease progression in chronic hepatitis C: modifiable and nonmodifiable factors. Gastroenterology 2008;134(6):1699-1714. DOI: 10.1053/j.gastro.2008.02.069.

4. Raimondi S, Bruno S, Mondelli MU, et al. Hepatitis C virus genotype $1 \mathrm{~b}$ as a risk factor for hepatocellular carcinoma development: a meta-analysis. J Hepatol 2009;50(6):1142-1154. DOI: 10.1016/ j.jhep.2009.01.019.

5. Probst A, Dang T, Bochud M, et al. Role of hepatitis C virus genotype 3 in liver fibrosis progression--a systematic review and meta-analysis. J Viral Hepat 2011;18(11):745-759. DOI: 10.1111/j.1365-2893.2011. 01481.x.

6. Kanwal F, Kramer JR, llyas J, et al. HCV genotype 3 is associated with an increased risk of cirrhosis and hepatocellular cancer in a national sample of U.S. Veterans with HCV. Hepatology 2014;60(1):98-105. DOI: 10.1002/hep.27095.
7. Castera L. Non-invasive assessment of liver fibrosis in chronic hepatitis C. Hepatol Int 2011;5(2):625-634. DOI: 10.1007/s12072-010-9240-0.

8. Castéra L, Vergniol J, Foucher J, et al. Prospective comparison of transient elastography, Fibrotest, APRI, and liver biopsy for the assessment of fibrosis in chronic hepatitis C. Gastroenterology 2005;128(2):343-350. DOI: 10.1053/j.gastro.2004.11.018.

9. European Association for Study of Liver; Asociacion Latinoamericana para el Estudio del Higado. EASL-ALEH Clinical Practice Guidelines: non-invasive tests for evaluation of liver disease severity and prognosis. J Hepatol 2015;63(1):237-264. DOI: 10.1016/ j.jhep.2015.04.006.

10. Colli A, Fraquelli $M$, Andreoletti $M$, et al. Severe liver fibrosis or cirrhosis: accuracy of US for detection-analysis of 300 cases. Radiology 2003;227(1):89-94. DOI: 10.1148/radiol.2272020193.

11. Yeom SK, Lee $\mathrm{CH}$, Cha SH, et al. Prediction of liver cirrhosis, using diagnostic imaging tools. World J Hepatol 2015;7(17):2069-2079. DOI: 10.4254/wjh.v7.i17.2069.

12. Lehmann M, Meyer MF, Monazahian M, et al. High rate of spontaneous clearance of acute hepatitis C virus genotype 3 infection. J Med Virol 2004;73(3):387-391. DOI: 10.1002/jmv.20103.

13. Mitchell AM, Stone AE, Cheng L, et al. Transmitted/founder hepatitis $C$ viruses induce cell-type- and genotype-specific differences in innate signaling within the liver. mBio 2015;6(2):e02510. DOI: 10.1128/ mBio.02510-14.

14. Negro F. Hepatitis $C$ virus-induced steatosis: an overview. Dig Dis 2010;28(1):294-299. DOI: 10.1159/000282105

15. Ryan MC, Desmond PV, Slavin JL, et al. Expression of genes involved in lipogenesis is not increased in patients with HCV genotype 3 in human liver. J Viral Hepat 2011;18(1):53-60. DOI: 10.1111/j.13652893.2010.01283.x.

16. Poynard T, Ratziu V, McHutchison J, et al. Effect of treatment with peginterferon or interferon alfa- $2 \mathrm{~b}$ and ribavirin on steatosis in patients infected with hepatitis C. Hepatology 2003;38(1):75-85. DOI: 10.1053/jhep.2003.50267.

17. Kumar D, Farrell GC, Fung C, et al. Hepatitis $C$ virus genotype 3 is cytopathic to hepatocytes: reversal of hepatic steatosis after sustained therapeutic response. Hepatology 2002;36(5):1266-1272. DOI: 10.1053/jhep.2002.36370.

18. Rubbia-Brandt L, Quadri R, Abid K, et al. Hepatocyte steatosis is a cytopathic effect of hepatitis $C$ virus genotype 3 . J Hepatol 2000;33(1):106-115. DOI: 10.1016/S0168-8278(00)80166-X.

19. Bochud P-Y, Cai T, Overbeck K, et al. Genotype 3 is associated with accelerated fibrosis progression in chronic hepatitis C. J Hepatol 2009;51(4):655-666. DOI: 10.1016/j.jhep.2009.05.016.

20. De Nicola S, Aghemo A, Rumi MG, et al. HCV genotype 3: an independent predictor of fibrosis progression in chronic hepatitis C. J Hepatol 2009;51(5):964-966. DOI: 10.1016/j.jhep.2009.08.001. 